\title{
MICRO MOLDED PDMS STRUCTURES FOR CARDIAC MYOCYTE RESTORING FORCE MEASUREMENTS
}

\author{
Yi Zhao, Hui Yu, Biao Li, and Xin Zhang \\ Department of Manufacturing Engineering and Fraunhofer USA Center for Manufacturing Innovation, \\ Boston University, Boston, Massachusetts 02215, USA (Tel: 617-358-2702; Email: xinz@bu.edu )
}

\begin{abstract}
This paper presents restoring force measurements of cardiac myocyte using micro molded PDMS structures. Various aspect ratios of the structures were successfully realized by changing precuring time for crosslinking. The calcium-activated force was derived from averaged displacement of the micro structures, with nano-newton resolution. This approach has the physiology and pathophysiology potential to be a basis for precise determination of restoring force in cardiovascular study.
\end{abstract}

\section{INTRODUCTION}

Cardiac myocyte, the most heavily worked muscle cell in living body, realizes contractile performance according to ventricular load. The calcium-activated isometric restoring force of cardiac myocyte in response to membrane action potential is critical to maintain heart behavior. Even subtle changes in restoring force can cause serious heart disease, which would not cause any noticeable consequences in other tissues.

To determine the restoring force, Velden et al. attached the isolated cardiac myocyte with silicon glue to a sensitive force transducer and a piezoelectric motor [1]. The attachment, however, induces an undesirable external stimulation to the myocyte, and there is an obvious trade-off between transducer sensitivity, which affects noise and drift, and compliance. To minimize the external stimulation to myocyte, Helmes et al. demonstrated an in vitro assay to assess the restoring force by measuring the velocity of recoil in unloaded myocyte after rigor-induced contraction of the force-generated unit (sarcomere) [2]. Although the surrogate measurement of restoring force from dynamics sarcomere relengthening was theoretically feasible, a measurement technique is needed for direct force probing. Consequently, investigators have relied on soft materials where the crosslinked chemistry was used to control the mechanical compliance $[3,4]$. Contraction generated deformation was traced by the displacement of beads or microfabricated markers embedded in the substrate, and restoring force can thus be derived. With important advances it made, this approach is, however, limited by the fact that it requires intensive computation of force calibration from continuous substrate deformation.

In this paper, we demonstrate a novel approach for restoring force measurements in isolated myocyte using closely spaced micro molded PDMS structures. This approach fairly isolates force probing from deformation effect of continuous substrate, which allows more accurate probing of restoring forces. With a nanonewton resolution, it has the physiology and pathophysiology potential to be a basis for precise determination of restoring force in cardiovascular study.

\section{FABRICATION}

Micro molded PDMS structures were fabricated from silicon molds as illustrated in Figure 1. To make a silicon template containing an array of single spaced holes or walls, silicon wafers coated with photoresist were exposed and developed (Figure 1a), followed by a deep RIE process for high aspect ratio structures (2$5 \mu \mathrm{m}$ in lateral dimension) (Figure 1b). Prepolymer PDMS
(Sylgard 184 with mixing ratio of 10:1) was poured on a glass slide and pre-cured at $65^{\circ} \mathrm{C}$ (Figure 1c). Then it was put in contact with the silicon template and pumped in vacuum chamber for 5-min degassing. Further curing was subsequently carried out at $65^{\circ} \mathrm{C}$ for fully crosslinking, making the total curing time up to 1 hour or so. Afterwards, the template was carefully peeled off from PDMS, with the pattern transferred (Figure 1d). It was found that higher aspect ratio associates with smaller pre-curing time and vice versa (Figure 1e), which is believed due to compromise between vacuum intensity and PDMS viscosity. Hence, resulted PDMS structures (Figure 2) are typically shorter in height as compared to their silicon templates. For the mixing ratio used in this work, i.e. 10:1, preferable structures with aspect ratio from 1:3 to 1:4 experience pre-curing time varied from 6 to 9 minutes. As shown in Figure 3, shorter curing causes structures leaning against their neighboring peers (left most); while longer curing compromises with lower force probing sensitivity (two in right most). The elastic modulus of the PDMS structures measured using the Hysitron Triboindenter system yields about $1000 \mathrm{kPa}$. (a)

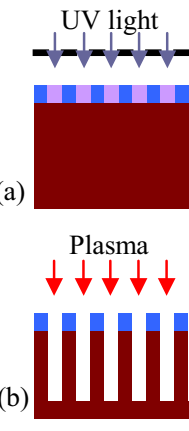

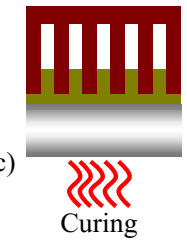

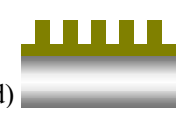

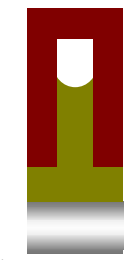

Short pre-curing Long pre-curing (e) (e)

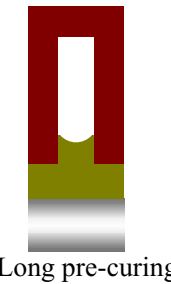

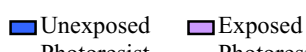
Photoresist Photoresist

Figure 1. Micro molding process for PDMS structures.

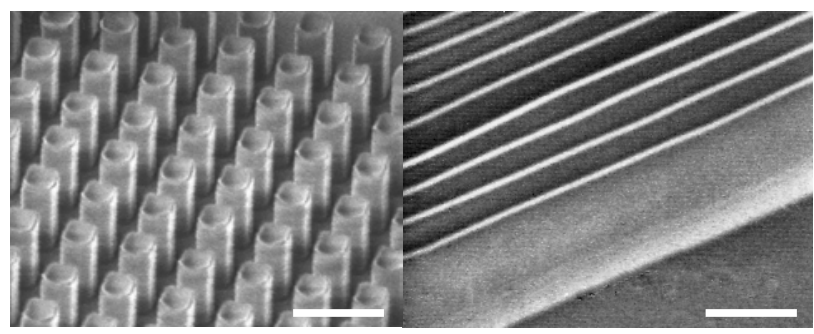

Figure 2. Micro molded PDMS pillars (a) and walls (b). (Scale bars indicate $10 \mu \mathrm{m})$.

\section{EXPERIMENTS AND RESULTS}

Fabricated PDMS structure peeled off from glass slide was immerged in medical ethanol for sterilization. Leminin was then coated and kept for 2 hours to enhance adhesion. The heart of Wistar rat was digested with collagenase via langendorf perfusion to obtain isolated, calcium tolerant myocytes. The myocytes were plated on laminin-coated PDMS structure. And the structure with the plated myocytes was put in culture media in a 100 petri dish and stored overnight in a $37^{\circ} \mathrm{C}$ incubator.

The experimental apparatus used to measure the structure displacement is described as follows. The PDMS substrate with myocytes was placed in a custom-made chamber, which was

Travel support has been generously provided by the Tranducers Research Foundation and by the DARPA MEMS and DARPA BioFlips programs 


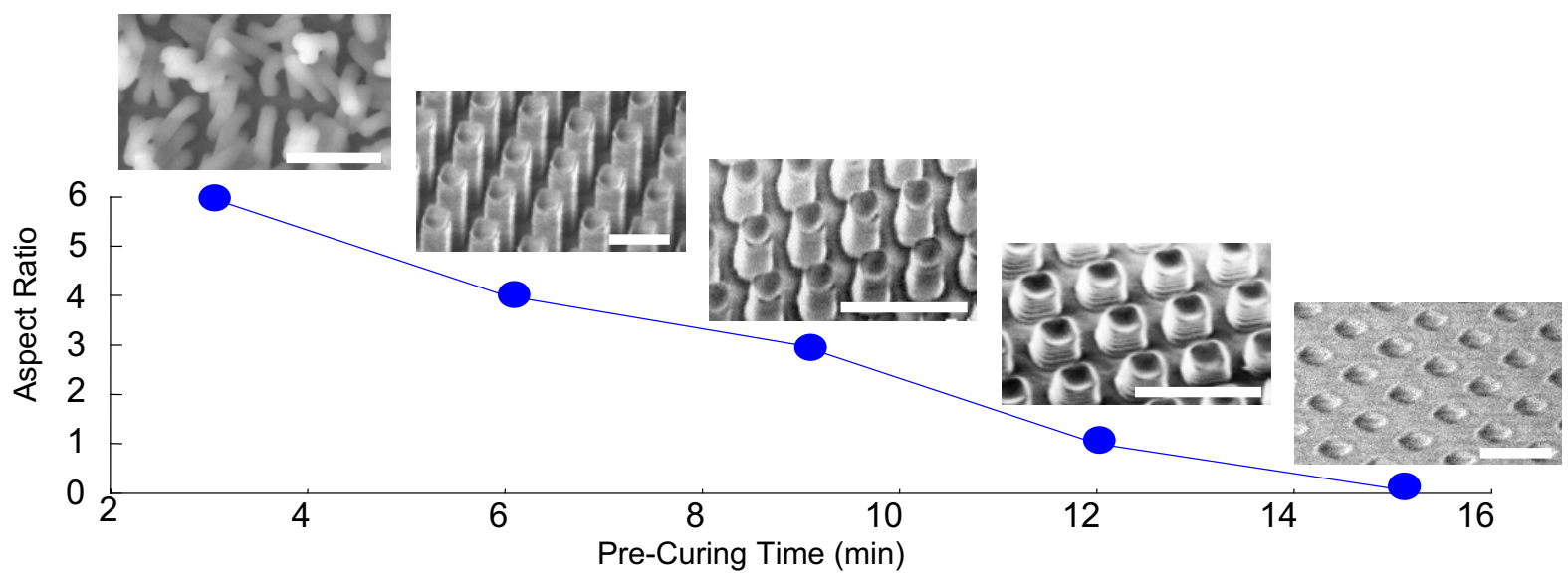

Figure 3. PDMS structures with various aspect ratios are realized with different pre-curing time. (Space bars indicate $10 \mu \mathrm{m}$ ) mounted on an inverted microscope $(400 \times)$, and stimulated by a digital pacer with periodical voltage pulse. The myocyte contracts in response to the pulse due to the raise of calcium level, followed by a passive relengthening. The process was imaged using a variable frame rate $(60-240 \mathrm{~Hz}) \mathrm{CCD}$ camera, and the images were digitized. The video lines were averaged vertically, and the program performed a Fast-fourier transform (FFT) on the averaged density trace, the peak of the resulting power spectra for various regions in a PDMS structure was thus obtained. The spectra peak represents the structure spacing, which is ultimately converted to the structure displacement. Contraction of the myocyte was traced in the same manner by changing focus to its top surface.

As one of typical results illustrated in Figure 4, the myocyte has an averaged contraction of $8.83 \mu \mathrm{m}$ (region A) in response to voltage pulse (indicated by black arrows), while the induced displacements of underneath pillars average $0.63 \mu \mathrm{m}$ (region B). The restoring force can thus be derived, providing Young's modulus and geometry of the pillars. The force probing resolution was calculated as about $2 \mathrm{nN}$. Furthermore, it was found that the pillars next to the myocyte (region C) do not represent an observable displacement in response to membrane action potential, indicating a fairly good isolation of the restoring force probing from deformation effect of continuous substrate.

Moreover, the non-sliding assumption between myocyte and its underneath PDMS pillars was validated using environmental

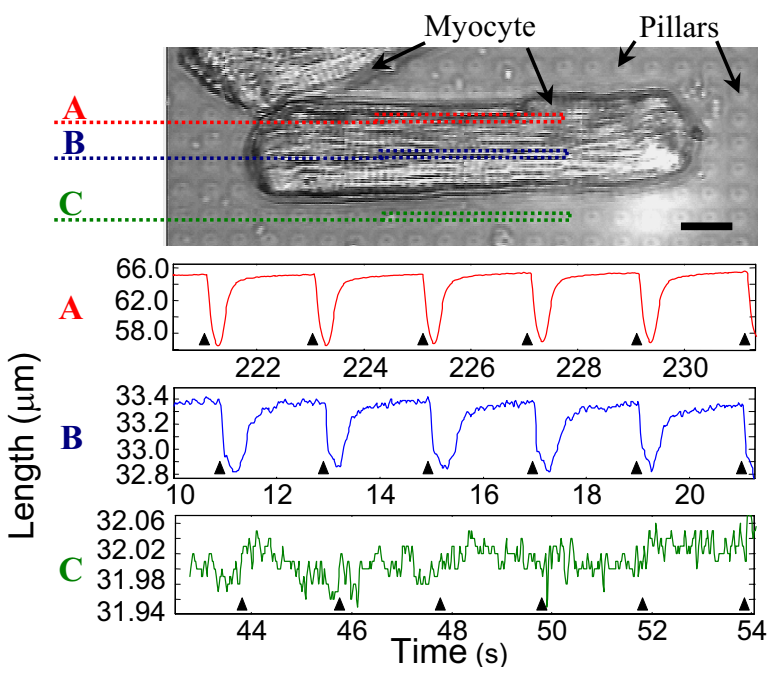

Figure 4. Tracings were carried out on myocyte surface (region $A)$, pillars underneath the myocyte (region B), and pillars next to the myocyte (region $C$ ), respectively. (Scale bar indicates $5 \mu \mathrm{m}$ )

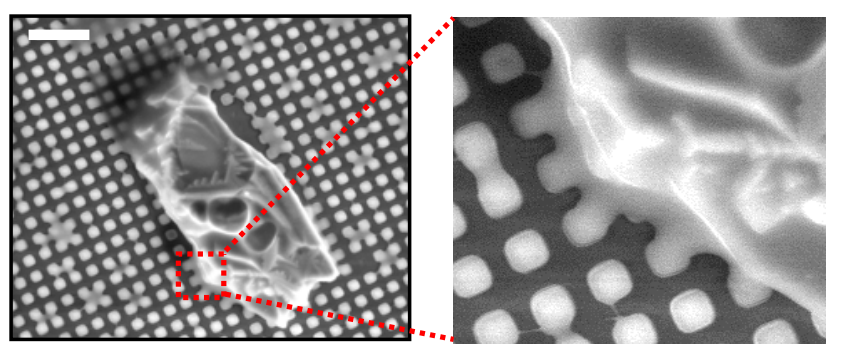

Figure 5. Environmental SEM observation after force probing indicates good adhesion of myocyte with underneath PDMS pillar arrays. (Scale bar indicates $10 \mu \mathrm{m}$ )

SEM after force probing (Figure 5). The good adhesion of the myocyte body with its underneath micro structure ensures the authenticity of the force measurements.

\section{CONCLUSION}

In this paper, we have demonstrated a novel approach for myocyte restoring force measurements using closely spaced micro molded PDMS structures. This method not only eliminates direct attachment of myocyte with force transducer, but also isolates force probing from continuous substrate deformation. With nanonewton resolution, it promises the potential with physiology and pathophysiology significance for precise determination of restoring force in cardiovascular study.

\section{ACKNOWLEDGEMENTS}

This work is supported by the NSF CAREER Award. The authors would like to thank Dr. Michiel Helmes and his colleagues at Whitaker Cardiovascular Institute, Boston University School of Medicine for their technical advices, and Dr. Richard Schalek at Harvard University Imaging Center for the assistance on environmental SEM observations.

\section{REFERENCES}

1. J. Velden, et al., "Force production in mechanically isolated cardiac myocyte from human ventricular muscle tissue", Cardiovascular Research, 28, 414 (1998).

2. M. Helmes, et al., "Time determines the Frank-Starling relation in early diastole", Journal of General Physiology, 121, 97 (2003).

3. A. Harris, et al., "Silicone rubber substrata: a new wrinkle in the study of cell locomotion", Science, 208, 177 (1980).

4. N. Balaban, et al., "Force and adhesion assembly: a close relationship between studies using elastic micropatterned substrates", Nature Cell Biology, 3, 466 (2001). 